\title{
Влияние температуры на энтальпию обмена разнозарядных ионов на полиметакриловом катионите
}

\author{
Карпюк Е.А., Гавлина О.Т., Иванов В.А., Каргов С.И. \\ Московский государственный университет имени М.В. Ломоносова, Москва
}

Поступила в редакцию 21.04.2017 г.

\begin{abstract}
Изучено влияние температуры на селективность и энтальпию обмена ионов $\mathrm{Ni}^{2+}-\mathrm{Na}^{+}$и $\mathrm{Mg}^{2+}$ - $\mathrm{Na}^{+}$на полиметакриловом катионите КБ-4П2 в интервале от 298 до 413 К. Показано, что при повышении температуры одновременно со значительным увеличением селективности к двухзарядному иону линейно увеличивается дифференциальная энтальпия. Наиболее сильно селективность возрастает в области «перегретых» растворов с температурой выше 373 К. Влияние ионного состава ионита на дифференциальную энтальпию значительно меньше, чем влияние температуры.
\end{abstract}

Ключевые слова: ионный обмен, полиметакриловый катионит, термодинамика, константа равновесия, термодинамические функции, дифференциальная энтальпия.

\section{The effect of temperature on the enthalpy of exchange of divalent - monovalent ions on a polymethacrylic cation exchange resin}

\author{
Karpyuk E.A., Gavlina O.T., Ivanov V.A., Kargov S.I. \\ M.V. Lomonosov Moscow State University, Moscow
}

The influence of temperature on the selectivity and enthalpy of the exchange of $\mathrm{Ni}^{2+}-\mathrm{Na}^{+}$and $\mathrm{Mg}^{2+}$ - $\mathrm{Na}^{+}$ions on the polymethacrylic cation exchange KB-4P2 in the range from 298 to $413 \mathrm{~K}$ was studied. It is shown that when the temperature is raised simultaneously with a significant increase in the selectivity to the doubly charged ion, the differential enthalpy increases linearly. The most strongly selectivity increases in the region of "superheated" solutions with a temperature above $373 \mathrm{~K}$. The differential enthalpy of the exchange of $\mathrm{Ni}^{2+}-\mathrm{Na}^{+}$ions increases more significantly than in the case of the exchange of $\mathrm{Ca}^{2+}-\mathrm{Na}^{+}$ions. Thus, in the exchange of calcium and sodium ions in the range from $273 \mathrm{~K}$ to $400 \mathrm{~K}$, the differential enthalpy increased from 4-6 kJ/eqv to $22-28 \mathrm{~kJ} / \mathrm{eqv}$, and in the exchange of $\mathrm{Ni}^{2+}-\mathrm{Na}^{+}$ions in the same temperature range ranges from 4-6 kJ/eqv to 28-34 kJ/eqv. In the case of the exchange of $\mathrm{Mg}^{2+}-\mathrm{Na}^{+}$ions, the values of the differential enthalpy were lower. The effect of the ionic composition of ion exchanger on the differential enthalpy is much less than the effect of temperature. The discovered properties of polymethacrylic cation exchanger can be of great importance for the development of ion-exchange separation processes, in particular, they show the possibility of using "superheated" solutions with a temperature above $373 \mathrm{~K}$ in dual-temperature reagentless processes.

Keywords: ion exchange, polymethacrylic resin, thermodynamics, equilibrium constant, thermodynamic functions, differential enthalpy.

\section{Введение}

В хроматографии в последние годы возникло новое направление, связанное с использованием «перегретой воды» («superheated water») с температурой выше 
373 К) в качестве элюента [1-3]. При повышении температуры диэлектрическая проницаемость воды значительно снижается, так что при температурах выше $373 \mathrm{~K}$ свойства воды приближаются к свойствам некоторых органических жидкостей или водно-органических растворов. Поэтому простым повышением температуры воды или водных растворов в хроматографии можно добиваться тех же эффектов, что и при использовании органических жидкостей в качестве элюирующих растворов. В этих работах обратили внимание и на то, что при столь значительном повышении температуры могут значительно изменяться ионообменные свойства используемых сорбентов.

Влиянию температуры на равновесие ионного обмена и ранее уделяли достаточное большое внимание. С использованием этого влияния удалось создать ряд процессов разделения, в которых не используются вспомогательные реактивы. Еще в 1950-х годах в Австралии был предложен и разработан впечатляющий двухтемпературный процесс частичного обессоливания слабосоленых природных вод [4, 5]. Значительно более поздние исследования в этой области привели к созданию и ряда других двухтемпературных процессов разделения, таких как безреагентные процессы очистки концентрированных растворов солей щелочных металлов от примесей двухзарядных ионов $[6,7]$, разделения солей щелочных металлов $[8,9]$, концентрирования калия из морской воды на природном цеолите с целью получения калийного удобрения [10-13], концентрирования бромида из морской воды [11] и иодида из природных рассолов $[14,15]$, извлечения тиоцианатов из растворов [16] и других. Обзоры этих работ и теоретические вопросы двухтемпературного разделения представлены в [17-20]. Эти процессы основаны на том, что сорбцию интересующих компонентов из растворов проводят при температуре, обеспечивающей повышенную селективность, а вытеснение из ионообменника достигают все тем же исходным раствором, но при иной температуре, обеспечивающей пониженную селективность. Важно отметить, что во всех этих процессах, также как и при исследовании влияния температуры на ионообменные свойства материалов, ограничивались верхним пределом температуры, не превышающим 373 К.

Анализ литературы по влиянию температуры на ионный обмен показывает, что в большинстве случаев оперируют средней величиной изменения энтальпии реакции ионного обмена для некоторого температурного интервала. В ряде работ это обосновано тем, что зависимость логарифма константы ионного обмена или коэффициента равновесия от обратной температуры оказывалась линейной [21-25]. Но в очень многих случаях авторы не утруждают себя рассмотрением такой зависимости, а просто рассчитывают среднее значение энтальпии.

В то же время еще в классических работах Боннера [26-28] было показано, что при обмене ионов натрия, аммония, меди и таллия на ион водорода, а также в некоторых случаях взаимного обмена двухвалентных ионов на сульфокатионитах с $8 \%$ и 16\% дивинилбензола (DVB) в интервале температур от 273 до 373 К зависимости логарифма константы равновесия от обратной температуры нелинейные и стандартные изменения энтальпии непостоянны. Изменение энтальпии ионного обмена с температурой было найдено и в работах $[29,30]$ для обмена ряда катионов в следовых количествах на $\mathrm{H}^{+}$и $\mathrm{Na}^{+}$формах сульфокатионита с $12 \%$ DVB и для обмена следовых количеств $\mathrm{Br}^{-}$на $\mathrm{Cl}^{-}$-форме сильноосновного анионита с 10\% DVB.

В работах $[2,3]$ влияние температуры на ионообменную селективность исследовали в связи с использованием “перегретой воды” в ионообменной хроматографии и также отметили непостоянство энтальпии при обмене ионов щелочных и щелочноземельных металлов на сульфокислотных ионообменниках.

Карпюк и др. / Сорбционные и хроматографические процессы. 2017. Т. 17. № 3 
Ранее мы обнаружили значительный рост энтальпии реакции ионного обмена с температурой для ионов кальция и натрия на некоторых промышленных полиметакриловых и полиакриловых катионитах $[31,32]$. Причем часть этих данных относятся к области «перегретых» растворов с температурой выше 373 К. В настоящей работе представим новые данные по влиянию температуры на энтальпию реакции ионного обмена ионов $\mathrm{Ni}^{2+}-\mathrm{Na}^{+}$и $\mathrm{Mg}^{2+}-\mathrm{Na}^{+}$. Предварительно кратко дадим необходимые сведения в области термодинамики ионного обмена.

\section{Теоретическая часть}

Термодинамика ионного обмена

Будем пользоваться терминологией, рекомендованной в [33]. При обмене ионов А и В с зарядами $z_{\mathrm{A}}$ и $z_{\mathrm{B}}$ на ионите

$$
\frac{1}{z_{A}} \bar{A}^{z_{A}}+\frac{1}{z_{B}} B^{z_{B}}=\frac{1}{z_{A}} A^{z_{A}}+\frac{1}{z_{B}} \bar{B}^{z_{B}}
$$

термодинамическая константа равновесия

$$
K=\frac{\bar{a}_{B}^{1 / z_{B}}}{\bar{a}_{A}^{1 / z_{A}}} \cdot \frac{a_{A}^{1 / z_{A}}}{a_{B}^{1 / z_{B}}}
$$

связана со стандартными значениями изменений энергии Гиббса $\Delta G^{O}$ и энтальпии $\Delta H^{o}$ реакции ионного обмена (1) по уравнениям [34]

$$
\begin{gathered}
R T \ln K=-\frac{1}{z_{B}} \bar{\mu}_{B}^{o}-\frac{1}{z_{A}} \bar{\mu}_{A}^{o}+\frac{1}{z_{A}} \mu_{A}^{o}-\frac{1}{z_{B}} \mu_{B}^{o}=-\Delta G^{o}, \\
\left(\frac{\partial \ln K}{\partial T}\right)_{p}=\frac{\Delta H^{o}}{R T^{2}}
\end{gathered}
$$

Здесь и далее $\bar{a}_{i}, a_{i}$ и $\bar{\mu}_{i}^{\mathrm{o}}, \mu_{i}^{\mathrm{o}}$ - активности и стандартные химические потенциалы компонентов в ионите и в растворе, соответственно; чертой обозначена принадлежность ионов и соответствующих параметров к фазе ионита. Стандартные величины $\Delta G^{\circ}$ и $\Delta H^{o}$ характеризуют замещение эквивалента ионов $\mathrm{A}$, содержащихся в ионите, на эквивалент ионов В из раствора, когда ионы А и В и в ионите, и в растворе находятся в стандартных состояниях. Если в качестве стандартных состояний ионов в ионите выбирают его моноионные формы, тогда эти величины интегральным образом характеризуют полный перевод ионообменника из формы иона А в форму иона В. При таком термодинамическом анализе ионообменных равновесий в качестве концентраций следует использовать молярности, моляльности или полные мольные доли обмениваемых ионов с учетом всех веществ, включая растворитель и сорбированные вещества [33].

Как показано в [35], исправленный коэффициент равновесия ионного обмена

$$
\widetilde{K}=\frac{\bar{c}_{B}^{1 / z_{B}}}{\bar{c}_{A}^{1 / z_{A}}} \cdot \frac{a_{A}^{1 / z_{A}}}{a_{B}^{1 / z_{B}}}
$$

по соотношению

$$
R T \ln \widetilde{K}=-\Delta G_{\bar{n}}+R T \ln \left(\bar{c}_{B}^{1 / z_{B}} / \bar{c}_{A}^{1 / z_{A}}\right),
$$

связан с дифференциальной энергией Гиббса

$$
\Delta G_{\bar{n}}=\frac{1}{z_{B}} \bar{\mu}_{B}-\frac{1}{z_{A}} \bar{\mu}_{A}+\frac{1}{z_{A}} \mu_{A}^{o}-\frac{1}{z_{B}} \mu_{B}^{o}=R T \ln \left(a_{B}^{1 / z_{B}} / a_{A}^{1 / z_{A}}\right)
$$


где $\bar{c}_{A}$ и $\bar{c}_{B}$ - концентрации обменивающихся ионов в ионообменнике.

Моляльности, молярности и полные мольные доли обменивающихся ионов в ионообменнике экспериментально определяются сложнее (а значит и с большими погрешностями), чем их эквивалентные доли. Однако при изучении дифференциальных термодинамических величин можно пользоваться и шкалой эквивалентных долей обмениваемых ионов в ионообменнике. Запишем коэффициент равновесия, аналогичный (5), в виде

$$
\widetilde{k}=\frac{y_{B}^{1 / z_{B}}}{y_{A}^{1 / z_{A}}} \cdot \frac{a_{A}^{1 / z_{A}}}{a_{B}^{1 / z_{B}}}
$$

$\left(y_{i}=z_{i} \bar{c}_{i} /\left(z_{A} \bar{c}_{A}+z_{B} \bar{c}_{B}\right)-\right.$ эквивалентная доля иона $i$ доля в ионите) и его связь с константой равновесия

$$
\ln \tilde{k}=\ln K+\ln \frac{y_{B}^{1 / z_{B}}}{y_{A}^{1 / z_{A}}}+\ln \frac{\bar{a}_{A}^{1 / z_{A}}}{\bar{a}_{B}^{1 / z_{B}}}
$$

Проведем простые преобразования

$$
\begin{gathered}
R T \ln \tilde{k}=-\left(\frac{1}{z_{B}} \bar{\mu}_{B}^{o}-\frac{1}{z_{A}} \bar{\mu}_{A}^{o}-\frac{1}{z_{B}} \mu_{B}^{o}+\frac{1}{z_{A}} \bar{\mu}_{A}^{o}\right)+R T \ln \frac{y_{B}^{1 / z_{B}}}{y_{A}^{1 / z_{A}}}+R T \ln \frac{\bar{a}_{A}^{1 / z_{A}}}{\bar{a}_{B}^{1 / z_{B}}}= \\
=-\left(\frac{1}{z_{B}} \bar{\mu}_{B}-\frac{1}{z_{A}} \bar{\mu}_{A}-\frac{1}{z_{B}} \mu_{B}^{o}+\frac{1}{z_{A}} \bar{\mu}_{A}^{o}\right)+R T \ln \frac{y_{B}^{1 / z_{B}}}{y_{A}^{1 / z_{A}}}
\end{gathered}
$$

В результате получаем соотношение с той же величиной $\Delta G_{\bar{n}}$

$$
R T \ln \widetilde{k}=-\Delta G_{\bar{n}}+R T \ln \left(y_{B}^{1 / z_{B}} / y_{A}^{1 / z_{A}}\right),
$$

Во-первых, обратим внимание, что по общему виду уравнения (6) и (11) отличаются от соотношения (3). Во-вторых, величину $\Delta G_{\bar{n}}$ удобнее рассчитывать по соотношению (7) на основании активностей обмениваемых ионов в растворе, равновесном с ионитом некоторого определенного состава (характеризуется молярными концентрациями $\bar{c}_{A}$ и $\bar{c}_{B}$ или эквивалентными долями $y_{A}$ и $\left.y_{B}\right)$. Сами концентрации обмениваемых ионов в ионите в расчете $\Delta G_{\bar{n}}$ не участвуют, а необходимы лишь для представления состава ионита, к которому относится рассчитанное значение $\Delta G_{\bar{n}}$.

Дифференцированием (6) и (11) по температуре при постоянных давлении и составе ионита получают соотношения, аналогичные уравнению изобары ВантГоффа (4), [35, 36]

$$
\left(\frac{\partial \ln \widetilde{K}}{\partial T}\right)_{p, \bar{n}}=\left(\frac{\partial \ln \tilde{k}}{\partial T}\right)=\frac{\Delta H_{\bar{n}}}{R T^{2}}
$$

где $\Delta H_{\bar{n}}=\frac{1}{z_{B}} \bar{h}_{B}-\frac{1}{z_{A}} \bar{h}_{A}-\frac{1}{z_{B}} h_{B}^{o}+\frac{1}{z_{A}} h_{A}^{o}-$ дифференциальное изменение энтальпии $\left(\bar{h}_{i}\right.$ - парциальная мольная энтальпия $i$-ого компонента, подстрочные индексы $p$ и $\bar{n}$ обозначают постоянство давления и чисел молей всех компонентов фазы). Необходимое для выполнения (12) условие постоянства состава ионита по всем компонентам при изменении температуры выполнить строго невозможно из-за того, что даже при фиксированном соотношении обменивающихся ионов его степень набухания и 
сорбция электролита зависят от температуры. Но в работах $[36,37]$ было показано, что влияние этого фактора сравнительно невелико даже для таких «термочувствительных» ионитов, как полиакриловые и полиметакриловые.

Дифференциальные термодинамические функции $\Delta G_{\bar{n}}$ и $\Delta H_{\bar{n}}$ относятся к иониту фиксированного по всем компонентам состава (при постоянных количествах молей обменивающихся ионов А и $\mathrm{B}$, воды, коионов $\mathrm{X}$ и фиксированных групп $\mathrm{R}$ ионита $\left.\bar{n}_{A}, \bar{n}_{B}, \bar{n}_{H_{2} O}, \bar{n}_{X}, \bar{n}_{R}\right)$. Они показывают изменения энергии Гиббса и энтальпии при замещении эквивалента ионов В из раствора на эквивалент иона А из бесконечно большого количества ионита определенного состава, когда ионы А и В в растворе находятся в стандартных состояниях. Это означает, что дифференциальные термодинамические функции $\Delta G_{\bar{n}}$ и $\Delta H_{\bar{n}}$ характеризуют обмен ионов на ионите с определенной степенью обмена и поэтому являются более информативными, чем стандартные величины, на что ранее неоднократно указывалось [34, 38].

Удобно использовать интегральные уравнения, получаемые из (12) при условии постоянства $\Delta H_{\bar{n}}$ в интервале температур $T_{1}-T_{2}$

$$
\Delta H_{\bar{n}}=\frac{R T_{1} T_{2}}{T_{2}-T_{1}} \ln \left(\frac{\widetilde{K}_{T_{2}}}{\widetilde{K}_{T_{1}}}\right)_{\bar{n}}=\frac{R T_{1} T_{2}}{T_{2}-T_{1}} \ln \left(\frac{\widetilde{k}_{T_{2}}}{\widetilde{k}_{T_{1}}}\right)_{\bar{n}}
$$

Но определение $\Delta H_{\bar{n}}$ по уравнению (13) осложняется высокой относительной погрешностью [39, 40]. При погрешностях величин $\widetilde{k}$ порядка 10-15\% и при $T_{2}-T_{1} \approx 20 \mathrm{~K}$ погрешность $\Delta H_{\bar{n}}$ в рассматриваемых системах может достигать десятков и даже сотен процентов. Высокая погрешность значительно снижает ценность получаемых таким образом величин энтальпии ионного обмена. Однако при нагревании суспензии селективного ионита и равновесного с ним раствора смеси электролитов, в котором прочнее сорбируемый ион является микрокомпонентом, в основном изменяется соотношение обменивающихся ионов в растворе, а ионный состав ионита изменяется очень слабо $[39,40]$. Такое изменение обычно меньше погрешности прямого аналитического определения состава ионита. Это означает, что дифференциальную энтальпию можно определить значительно точнее на основании анализа только состава раствора в равновесной системе при двух температурах по уравнению

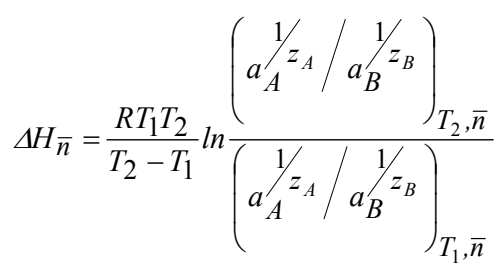

Изложенный подход использован в настоящей работе.

\section{Эксперимент}

Материалы. Подготовка ионитов. Исследовали гелевый карбоксильный полиметакриловый катионит КБ-4П2 с 2.5\% ДВБ (приблизительно 1 сшивка на 60 метакрилатных мономерных звеньев).

Ионит в смешанных $\mathrm{Mg}$, $\mathrm{Na}-$ и $\mathrm{Ni}, \mathrm{Na}$-формах с заданной долей двухвалентного иона $\mathrm{M}^{2+}\left(\mathrm{Mg}^{2+}\right.$ или $\left.\mathrm{Ni}^{2+}\right)$ готовили следующим образом. Суспензию ионита в $\mathrm{Na-форме} \mathrm{делили} \mathrm{на} \mathrm{две} \mathrm{порции} \mathrm{с} \mathrm{определенными} \mathrm{объемами.} \mathrm{Одну} \mathrm{из} \mathrm{них} \mathrm{помещали}$ в колонку и переводили в М-форму, пропуская избыток 0.5 н раствора $\mathrm{MCl}_{2}$. Ионит промывали небольшим количеством воды. После этого две порции ионита в Na- и 
М-формах вновь соединяли в колбе (используя для этого небольшое количество 2.5 н раствора $\mathrm{NaCl}$ ) и перемешивали в течение суток для установления одинакового ионного состава отдельных зерен.

Определение энтальпии ионного обмена. В колбу с суспензией ионита в смешанной M, Na- форме заданного состава (объем суспензии $200 \mathrm{~cm}^{3}$ ) добавляли 150$300 \mathrm{~cm}^{3}$ приготовленного смешанного 2.3-2.8 н раствора $\mathrm{NaCl}$ и $\mathrm{MCl}_{2}$, соответствующего равновесию с требуемым составом ионита при температуре $T_{1}$. Колбу помещали в термостат с температурой $T_{l}$, суспензию периодически перемешивали и анализировали концентрацию $\mathrm{M}^{2+}$ в растворе комплексонометрическим титрованием, чтобы убедиться в равновесности системы.

Затем температуру повышали на 10-25 К (до значения $T_{2}$ ), систему вновь приводили в равновесие и анализировали состав раствора до установления равновесия. Время достижения равновесия зависит от температуры и варьируется от 3 часов при температуре 298 К до нескольких минут при более высоких температурах. После установления равновесия в растворе также определяли концентрацию $\mathrm{Na}^{+}$методом эмиссионной пламенной фотометрии (убеждались, что концентрация $\mathrm{NaCl}$ в растворе изменялась незначительно). Далее температуру повышали и анализировали раствор последовательно еще несколько раз, достигая некоторую максимальную в условиях данного эксперимента температуру.

В некоторых из опытов температура была выше 373 К. В этих случаях толстостенную стеклянную колбу плотно закрывали тефлоновой крышкой с резиновой прокладкой с тефлоновым перекрывающимся капилляром, один из концов которого погружен в раствор внутри колбы (рис. 1). Давление в колбе повышалось за счет установления равновесного давления паров воды при закрытом кране и не позволяло раствору кипеть. При открывании крана повышенное давление обеспечивало истечение раствора через капилляр и отбор проб для анализа.

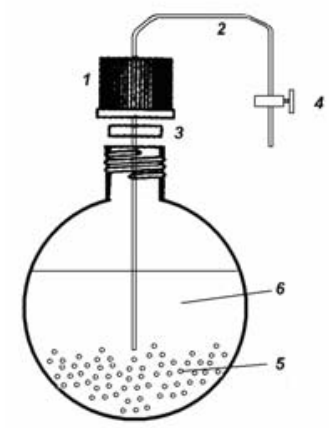

Рис. 1. Колба для изучения равновесия ионного обмена при температуре выше 373 К (1 - тефлоновая крышка, тефлоновый капилляр, 3 - резиновая прокладка, 4 - кран, 5 - ионообменник, 6 - раствор.

После этого проводили обратный эксперимент с последовательным понижением температуры от достигнутого в конце прямого эксперимента значения до начального значения через те же самые стадии.

В конце серии экспериментов ионит из колбы быстро перегружали в колонку, водоструйным насосом удаляли раствор из межзеренного пространства и через колонку пропускали избыток 0.5 н раствора $\mathrm{HCl}$. В фильтрате определяли количества вытесненных ионов $\mathrm{M}^{2+}$ и $\mathrm{Na}^{+}$.

При повышении температуры степень набухания полиметакриловых и полиакриловых катионитов несколько уменьшается (изменение объема ионита достигает $18-20 \%$ при изменении температуры от 293 до 363 К [36]) и происходит разбавление

Карпюк и др. / Сорбционные и хроматографические процессы. 2017. Т. 17. № 3 
раствора водой из фазы ионообменника. Однако при небольшом различии температур $T_{1}$ и $T_{2}$ порядка $20 \mathrm{~K}$ изменение концентрации раствора пренебрежимо мало.

Изменение состава ионита с температурой в описанных экспериментах рассчитывали по следующей формуле

$$
y_{\mathrm{M}}\left(T_{2}\right)=y_{\mathrm{M}}\left(T_{1}\right)+\frac{V \cdot\left(c_{\mathrm{M}}\left(T_{2}\right)-c_{\mathrm{M}}\left(T_{1}\right)\right)}{W \cdot \bar{c}_{\mathrm{o}}}
$$

где $W$ и $V$ - объем суспензии ионита и объем раствора в колбе $\left(\mathrm{cm}^{3}\right), \bar{c}_{o}-$ концентрация обменивающихся ионов в ионите (мг-экв/ $\mathrm{cm}^{3}$ суспензии), $c_{M}\left(T_{i}\right)-$ равновесная при температуре $T_{i}$ концентрация ионов М в растворе (мг-экв/ см${ }^{3}$ ). Оказалось, что во всех экспериментах при повышении температуры (как относительно небольшом на $10 \mathrm{~K}-20 \mathrm{~K}$, так и более широком $\sim 70 \mathrm{~K})$ состав ионита изменялся лишь на 0.5-2 \%. Такое изменение состава ионита нельзя надежно установить аналитическими методами. Можно считать, что в экспериментах хорошо выполнялись условия постоянства состава ионита, при которых допустимо использовать предложенную методику определения $\Delta H_{\bar{n}}$.

При расчетах коэффициента равновесия, дифференциальной энтальпии и изменения теплоёмкости реакции ионного обмена использовали соотношения:

$$
\begin{gathered}
\widetilde{\widetilde{k}}=\frac{y_{M}^{1 / 2}}{y_{N a}} \cdot \frac{c_{N a}}{c_{M}^{1 / 2}} \\
\Delta H_{\bar{n}}=\frac{R T_{1} T_{2}}{T_{2}-T_{1}} \cdot\left[\ln \frac{\left(c_{N a} / c_{M}^{1 / 2}\right)_{T_{2}}}{\left(c_{N a} / c_{M}^{1 / 2}\right)_{T_{1}}}\right]_{\bar{n}} \\
\Delta C_{p, \bar{n}}=\left(\frac{\partial \Delta H_{\bar{n}}}{\partial T}\right)_{p}
\end{gathered}
$$

В этих соотношениях $y_{\mathrm{M}}$ и $y_{\mathrm{Na}}-$ эквивалентные доли ионов $\mathrm{M}^{2+}$ и $\mathrm{Na}^{+}$в ионите, $c_{\mathrm{M}}$ и $c_{\mathrm{Na}}-$ молярные концентрации ионов в растворе.

Использование молярных концентраций связано с тем, что при записи выражения для химического потенциала иона, использованного при выводе этих соотношений, в качестве стандартного состояния выбран раствор с концентрацией 1 моль/дм ${ }^{3}$ [40]. Но рассчитываемая величина $\Delta H_{\bar{n}}$ имеет размерность кДж/г-экв в соответствии с записью реакции ионного обмена, которой соответствуют эти соотношения.

При определении энтальпии коэффициенты активности не учитывали, т.к. данные по их температурным зависимостям для исследуемых смешанных растворов $\mathrm{NaCl}-\mathrm{NiCl}_{2}$ и NaCl-MgCl 2 в литературе отсутствуют. Но ранее при исследовании системы NaCl-CaCl 2 было показано [32], что вклад температурных зависимостей коэффициентов активности в величину $\Delta H_{\bar{n}}$ очень мал.

\section{Обсуждение результатов}

На рис. 2 представлена зависимость коэффициента равновесия обмена ионов $\mathrm{Ni}^{2+}-\mathrm{Na}^{+}$и $\mathrm{Mg}^{2+}-\mathrm{Na}^{+}$от температуры при одинаковой доле двухзарядного иона в ионите, равной 0.45 . В первой системе влияние температуры оказалось несколько более значительным, чем в изученной ранее системе $\mathrm{Ca}^{2+}-\mathrm{Na}^{+}[32]$, а во второй на- 
оборот более слабым. Наиболее сильно селективность возрастает в области «перегретых» растворов с температурой выше $373 \mathrm{~K}$.

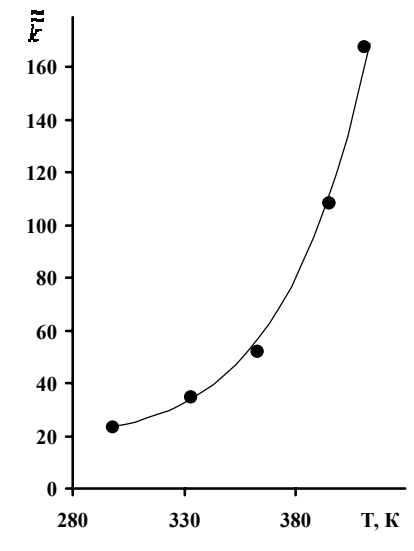

a

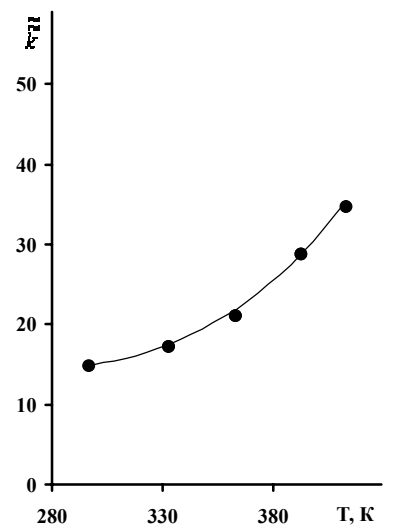

6

Рис. 2. Зависимость коэффициента равновесия обмена ионов $\mathrm{Ni}^{2+}-\mathrm{Na}^{+}$(a), $\mathrm{Mg}^{2+}-\mathrm{Na}^{+}$(б) от температуры при доле $\mathrm{M}^{+2}$ в ионите, равной 0.45 .

На рис. 3 представлены экспериментальные зависимости $\Delta H_{\bar{n}_{i}}$. Эти данные показали, что на полиметакриловом катионите с повышением температуры дифференциальная энтальпия обмена ионов $\mathrm{Ni}^{+2}-\mathrm{Na}^{+}$линейно возрастает более значительно, чем ионов $\mathrm{Ca}^{+2}-\mathrm{Na}^{+}$[32]. Так при обмене ионов кальция и натрия в интервале от $273 \mathrm{~K}$ до $400 \mathrm{~K}$ дифференциальная энтальпия возрастала от значений $4-6$ кДж/г-экв до 22-28 кДж/г-экв [32], а при обмене ионов $\mathrm{Ni}^{+2}-\mathrm{Na}^{+}$в том же температурном интервале - от 4-6 кДж/г-экв до 28-34 кДж/г-экв (без учета влияния температуры на коэффициенты активностей солей). Влияние ионного состава ионита на $\Delta H_{\bar{n}}$ значительно меньше, чем влияние температуры. В случае обмена ионов $\mathrm{Mg}^{+2}-$ $\mathrm{Na}^{+}$значения дифференциальной энтальпии оказались ниже, но и в этом случае $\Delta H_{\bar{n}}$ достаточно резко возрастала с температурой.

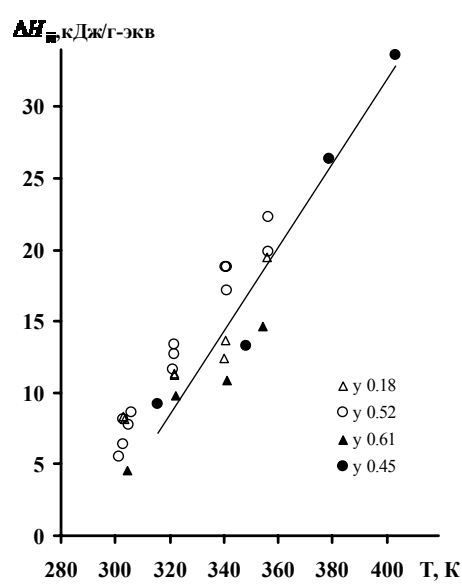

a

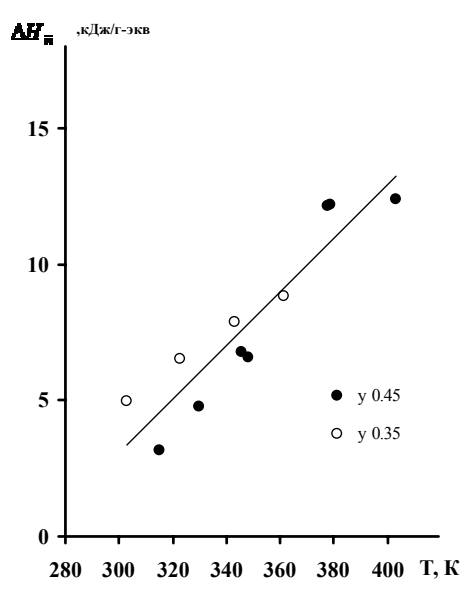

б

Рис. 3. Зависимости $\Delta H_{\bar{n}}$ от температуры при обмене ионов $\mathrm{Ni}^{2+}$ и $\mathrm{Na}^{+}$(а) и $\mathrm{Mg}^{2+}$ и $\mathrm{Na}^{+}$(б) на катионите КБ-4П2 (эквивалентная доля двухзарядного иона в ионите приведена на графиках).

Причины резкого возрастания с температурой селективности карбоксильных ионообменников при обмене однозарядных ионов на двухзарядные с температурой 
подробно рассматривались нами ранее [41] и были объяснены преобладающей ролью изменения гидратации обменивающихся ионов при переходе из одной фазы в другую. Здесь же акцентируем наше внимание на линейной зависимости $\Delta H_{\bar{n}}$ от температуры, которая свидетельствует о постоянстве величины $\Delta_{r} C_{p}$ (изменение теплоемкости реакции обмена разнозарядных ионов). Полученные значения $\Delta_{r} C_{p}$ представлены в таблице.

Таблица. Изменение теплоемкости реакции обмена разнозарядных ионов

\begin{tabular}{|c|c|c|c|}
\hline № & Система & Доля $\mathrm{M}^{+2}$ в ионите, $y_{M}$ & $\Delta_{r} C_{p}$, Дж/г-экв $\cdot$ К \\
\hline 1 & \multirow{4}{*}{$\mathrm{Ni}^{+2}-\mathrm{Na}^{+}$} & 0.18 & 200 \\
\hline 2 & & 0.44 & 290 \\
\hline 3 & & $0,51-52$ & 260 \\
\hline 4 & & $0,58-0,61$ & 180 \\
\hline 5 & \multirow{2}{*}{$\mathrm{Mg}^{+2}-\mathrm{Na}^{+}$} & 0,45 & 115 \\
\hline 6 & & 0,35 & 67 \\
\hline
\end{tabular}

\section{Заключение}

Проведенное исследование продемонстрировало, что при обмене ионов $\mathrm{Ni}^{2+}-$ $\mathrm{Na}^{+}$и $\mathrm{Mg}^{2+}-\mathrm{Na}^{+}$на полиметакриловом катионите КБ-4П2, также как и в случае системы $\mathrm{Ca}^{2+}-\mathrm{Na}^{+}$, повышение температуры (в интервале от 298 до 413 К) приводит к значительному возрастанию селективности к двухзарядному иону. Наиболее сильно селективность возрастает в области «перегретых» растворов с температурой выше 373 К. При повышении температуры одновременно с увеличением селективности к двухзарядному иону линейно увеличивается дифференциальная энтальпия (в 7 раз в первой системе и в 4 раза - во второй системе). Влияние ионного состава ионита на дифференциальную энтальпию значительно меньше, чем влияние температуры. Обнаруженные свойства полиметакрилового катионита могут иметь большое значение для развития процессов ионообменного разделения, в частности, они показывают возможность использования «перегретых» растворов с температурой выше $373 \mathrm{~K}$ в двухтемпературных безреагентных процессах.

\section{Список литературы}

1. Coym J.W., Dorsey J.G. // Analytical Letters. 2004. Vol. 37. No 5. pp. 1013-1023.

2. Masami Shibukawa, Tomomi Shimasaki, Shingo Saito, Takashi Yarita // Analytical Chemistry. 2009. Vol. 81. No 19. pp. 8025-8032.

3. Masami Shibukawa, Akihiko Taguchi, Yusuke Suzuki, Kazunori Saito et al. // Analyst. 2012. Vol. 137. pp. 3154-3159.

4. Wilhelm R.H., Rice A.W., Bendelius A.R. // Ind. Eng. Chem. Fundam. 1966. Vol. 5. No 1. pp. 141-144.

5. Bolto B.A., Weiss D.E. // In: Ion Exchange and Solvent Extraction. (Series of Advances). Vol. 7. Eds.: J.A.Marinsky and Y.Marcus. Marcel Dekker. Inc. New-York-Basel-Hong Kong. 1977. pp. 221-289.
6. Ivanov V., Timofeevskaya V., Gorshkov V. // Reactive and Functional Polymers. 1992. Vol. 17. No 1. pp. 101-107.

7. Gorshkov V., Ivanov V. // Solvent Extraction and Ion Exchange. 1999. Vol. 17. No 4. pp. 695-766.

8. Leavitt F.W. US Patent No 5681477 (1997).

9. Ivanov V.A, Timofeevskaja V.D, Gavlina O.T, Gorshkov V.I. // Microporous and Mesoporous Materials. 2003. Vol.65. No 2-3. pp. 257-265.

10.Хамизов Р.Х., Мелихов С.А., Новикова В.А., Сковыра В.В. Патент РФ № 2006495 (1994).

11.Khamizov R.Kh, Muraviev D.N., War- 
shawsky A. // In: Ion Exchange and Solvent Extraction. Series of Advances. Vol. 12. (Edited by J.A.Marinsky and Y.Marcus). New-YorkBasel-Hong Kong: Marcel Dekker. Inc. 1995. pp. 93-148.

12.Окуджава Н.Г., Беруашвили Ц. А., Мамукашвили Н.Ш. // Сорбиионные и хроматографические прочессы. 2008. Т. 8. №. 5. С. 875-880.

13.Гоциридзе Р.С., Испирян А.Г., Лория Л.И., Мепаришвили Н.А. и др. // Сорбиионные и хроматографические прочессы. 2009. T. 9. № 2. C. 254-260.

14.Ножов А.М., Кособрюхова О.М., Хамизов Р.Х. // Сорбиионные и хроматографические прочессы. 2003. Т. 3. № 2. С. 159-168.

15.Ножов А.М., Грачев А.С., Хамизов Р.Х. // Сорбиионные и хроматографические проuесcы. 2003. T. 3. № 3. С. 255-262

16.Крачак А.Н., Хамизов Р.Х., Кузьминова И.Г. // Сорбиионные и хроматографические проиессы. 2016. Т. 16. № 1. С. 8-15.

17. Grevillot G. // In: Handbook of heat and mass transfer. Gulf Publ., West Orange. N.Y. U.S.A. 1985. pp. 1283-1321.

18. Tondeur D., Grevillot G. // In: Ion exchange science and technol. NATO advanced study and inst. Troia. 1986. No 107. pp. 369399.

19. Khamizov R. Kh., Ivanov V.A., Tikhonov N.A. // In: Ion Exchange and Solvent Extraction: A Series of Advances. Vol. 20. Ed. Arup K. SenGupta. Taylor \& Francis Group, LLC. Boca Raton, FL: CRC Press. 2010. Ch. 5. 33. 171-232.

20.Khamizov R. Kh., Ivanov V.A., Madani A.A. // React. Func. Polym. 2010. Vol. 70. No 8. pp. 521-530.

21.Lokhande R. S., Singare P.U. // Asian J. Chem. 1998. Vol. 10. No 6. pp. 1026-1028.

22.Son W.-K., Kim S. H., Park S.-G. // Bull. Korean Chem. Soc. 2001. Vol. 22. No 1. pp. 5358.

23.Lee I.-H., Kuan Yu.-Ch, Chern J.-M. // J. Chinese Inst Chem. Eng. 2007. Vol. 38. No 1. pp. 71-84.

24. Millicent U., A. Okoye F.A., Akaranta O. // J. Am. Chem. Soc. 2012. Vol. 2, No 1. pp. 2537.

\section{References}

1. Coym J.W., Dorsey J.G., Analytical Letters., 2004, Vol. 37, No 5, pp. 1013-1023.

2. Masami Shibukawa, Tomomi Shimasaki,
25. Singare P. U., Patange A. N. // Int. Lett. Chem., Phys. Astr. 2014. Vol. 11. No 1. pp. 4450.

26.Bonner O.D., Smith L.L. // J . Phys. Chem. 1957. Vol. 61. No 12. pp. 1614-1617.

27.Bonner O.D., Pruett R.R. // J. Phys. Chem. 1959. Vol. 63. No 9. pp. 1417-1420.

28.Bonner O.D., Pruett R.R. // J. Phys. Chem. 1959. Vol. 63. No 9. pp. 1420-1423.

29.Kraus K.A., Raridon R.J. // J. Phys. Chem. 1959. Vol. 63. No 11. pp 1901-1907.

30.Kraus K.A., Raridon R.J., Holcomb D.L. // Chromatogr. 1960. Vol. 3. No 1. pp. 178-179.

31.Иванов В.А., Горшков В.И., Гавлина О.Т., Илюхина Е.А. // Журн. физ. химии. 2006. T.80. № 11. С. 2051-2057.

32.Иванов В.А., Тимофеевская В.Д., Горшков В.И., Гавлина О.Т. и др. // Журн. физ. химии. 2007. Т. 81. № 11. С. 1927-1931.

33.Харьюла Р., Лето Ю. // Журн. физ. химии. 1996. Т. 70. № 9. С. 1723-1725.

34.Soldatov V.S. // In: Ion Exchangers.(Konrad Dorfner, Ed.). Berlin, New York: De Gruyter. 1992. pp. 1243-1275.

35.Иванов В.А., Тимофеевская В.Д., Горшков В.И. // Журн. физ. химии. 2000. Т. 74. № 4. C. 730-733.

36.Иванов В.А., Тимофеевская В.Д., Горшков В.И., Дроздова Н.В. // Журн. физ. химии. 2000. T. 74, № 5. C. 917-920.

37.Karpyuk E.A., Titova O.I., Pastukhov A.V., Davankov V.A. et al. // Solvent Extraction and Ion Exchange. 2016. Vol. 34. No 4. pp. $362-374$

38.Солдатов В.С. Простые ионообменные равновесия. Наука и техника. Минск. 1972. $223 \mathrm{c}$.

39.Иванов В.А., Тимофеевская В.Д., Горшков В.И., Ярославцев А.А и др. // Сорбционные и хроматографические прочессы. 2004. T. 4. № 5. C. 550-560.

40.Иванов В.А., Горшков В.И., Гавлина О.Т., Илюхина Е.А. // Журн. физ. химии. 2007. T. 81. № 10. C. 1582-1585.

41.Ivanov V.A., Gorshkov V.I., Timofeevskaya V.D., Drozdova N.V. // Reactive and Functional Polymers. 1998. Vol. 38. No 2-3. pp. 205-218.

Shingo Saito, Takashi Yarita, Analytical Chemistry, 2009, Vol. 81, No 19, pp. 8025-8032.

3. Masami Shibukawa, Akihiko Taguchi, 
Yusuke Suzuki, Kazunori Saito et al., Analyst., 2012, Vol. 137, pp 3154-3159.

4. Wilhelm R.H., Rice A.W., Bendelius A.R., Ind. Eng. Chem. Fundam., 1966, Vol. 5, No 1, pp. 141-144.

5. Bolto B.A., Weiss D.E., In: Ion Exchange and Solvent Extraction. (Series of Advances). Vol.7. Eds.: J.A.Marinsky and Y.Marcus. Marcel Dekker, Inc. New-York-Basel-Hong Kong, 1977, pp. 221-289.

6. Ivanov V., Timofeevskaya V., Gorshkov V., Reactive and Functional Polymers, 1992, Vol. 17, No 1. pp. 101-107.

7. Gorshkov V., Ivanov V., Solvent Extraction and Ion Exchange, 1999, Vol. 17, No 4, pp. 695-766.

8. Leavitt F.W. US Patent No 5681477 (1997).

9. Ivanov V., Timofeevskaja V., Gavlina O., Gorshkov V., Microporous and Mesoporous Materials, 2003, Vol. 65, No 2-3, pp. 257-265.

10. Khamizov R., Melikhov S., Novikova V., Skovyra V. Patent of Russian Federation No 2006495 (1994).

11. Khamizov R.Kh, Muraviev D.N., Warshawsky A., In: Ion Exchange and Solvent Extraction. Series of Advances. Vol. 12. (Edited by J.A.Marinsky and Y.Marcus). New-YorkBasel-Hong Kong: Marcel Dekker, Inc. 1995, pp. 93-148.

12. Okudzhava N.G., Beruashvili C. A., Mamukashvili N.Sh., Sorbtsionnye i khromatografiheskie protsessy, 2008, Vol. 8, No 5, pp. 875880.

13. Gociridze R.S., Ispirjan A.G., Lorija L.I., Meparishvili N.A. et al., Sorbtsionnye i khromatograficheskie protsessy, 2009, Vol. 9, No 2, pp. 254-260.

14. Nozhov A.M., Kosobrjuhova O.M., Hamizov R.H., Sorbtsionnye $i$ khromatograficheskie protsessy, 2003, Vol. 3, No 2, pp. 159168.

15. Nozhov A.M., Grachev A.S., Hamizov R.H., Sorbtsionnye i khromatograficheskie protsessy, 2003, Vol. 3, No 3, pp. 255-262.

16. Krachak A.N., Hamizov R.H., Kuz'minova I.G., Sorbtsionnye $i$ khromatograficheskie protsessy, 2016, Vol. 16, No 1, pp. 8-15.

17. Grevillot G. Principles of parametric pumping, In: Handbook of heat and mass transfer. Gulf Publ., West Orange, N.Y., U.S.A. 1985, pp. 1283-1321.

18. Tondeur D., Grevillot G. In: Ion exchange science and technol. NATO advanced study and inst. Troia. 1986, No 107, pp. 369-
399.

19. Khamizov R. Kh., Ivanov V.A., Tikhonov N.A., In: Ion Exchange and Solvent Extraction: A Series of Advances, Vol. 20. Ed. Arup K. SenGupta. Taylor \& Francis Group, LLC. Boca Raton, FL: CRC Press.,2010, Ch. 5, pp. 171-232.

20. Khamizov R. Kh., Ivanov V.A., Madani A.A., React. Func. Polym., 2010, Vol. 70, No 8, pp. 521-530.

21. Lokhande R. S., Singare P.U., Asian J. Chem., 1998, Vol. 10, No 6, pp. 1026-1028.

22. Son W.-K., Kim S. H., Park S.-G., Bull. Korean Chem. Soc., 2001, Vol. 22, No 1, pp. 53-58.

23. Lee I.-H., Kuan Yu.-Ch, Chern J.-M., J. Chinese Inst Chem. Eng., 2007, Vol. 38, No 1, pp. 71-84.

24. Millicent U., A. Okoye F.A., Akaranta O., J. Am. Chem. Sci., 2012, Vol. 2, No 1, pp. 25-37.

25. Singare P.U., Patange A.N., Int. Lett. Chem., Phys. Astr., 2014, Vol. 11, No 1, pp. 4450.

26. Bonner O.D., Smith L.L., J. Phys. Chem., 1957, Vol. 61, No 12, pp. 1614-1617.

27. Bonner O.D., Pruett R.R., J. Phys. Chem., 1959, Vol. 63, No 9, pp. 1417-1420.

28. Bonner O.D., Pruett R.R., J. Phys. Chem., 1959, Vol. 63, No 9, pp. 1420-1423.

29. Kraus, K.A., Raridon, R.J., J. Phys. Chem., 1959, Vol. 63, No 11, pp 1901-1907.

30. Kraus K.A., Raridon R.J., Holcomb D.L., Chromatogr., 1960, Vol. 3, No 1, pp. 178-179.

31. Ivanov V.A., Gorshkov V.I., Gavlina O.T., Ilyukhina E.A., Russian Journal of Physical Chemistry, 2006, Vol. 80, No 11, pp. 18261831.

32. Ivanov V., Gavlina O., Ilyukhina E., Gorshkov V., Russian Journal of Physical Chemistry A, 2007, Vol. 81, No 11, pp. 17231727.

33. Harjula R., Lehto J., React. Func. Polymers, 1995, Vol. 27, pp. 147-153.

34. Soldatov V.S., In: Ion Exchangers.(Konrad Dorfner, Ed.). Berlin, New York: De Gruyter. 1992, pp. 1243-1275.

35. Ivanov V., Timofeevskaya V., Gorshkov V., Russian Journal of Physical Chemistry, 2000, Vol. 74, No 4, pp. 637-640.

36. Ivanov V.A., Timofeevskaya V.D., Gorshkov V.I., Drozdova N.V., Russian Journal of Physical Chemistry A, 2000, Vol. 74, No 5, pp. 812-815.

37. Karpyuk E.A., Titova O.I., Pastukhov 
A.V., Davankov V.A. et al., Solvent Extraction and Ion Exchange, 2016, Vol. 34, No 4, pp. 362-374.

38. Soldatov V.S. Prostye ionoobmennye ravnovesija. Nauka i tehnika, Minsk, 1972, $223 \mathrm{p}$.

39. Ivanov V.A, Timofeevskaja V.D., Gorshkov V.I., Jaroslavcev A.A. et al., Sorbtsionnye $i$ khromatograficheskie

Иванов Владимир Александрович - д.Х.н., профессор, химический факультет МГУ имени. М.В. Ломоносова, Москва, тел.: (495) 939-35-30

Карпюк Екатерина Анатольевна - к.х.н., доцент, химический факультет МГУ имени. М.В. Ломоносова, Москва

Гавлина Ольга Тихоновна - к.х.н., старший научный сотрудник, химический факультет МГУ имени. М.В. Ломоносова, Москва

Каргов Сергей Игоревич - д.Х.Н., професcop, химический факультет МГУ имени. М.В. Ломоносова, Москва. protsessy,2004, Vol. 4, No 5, pp. 550-560.

40. Ivanov V.A., Gorshkov V.I., Gavlina O.T., Ilyukhina E.A., Russian Journal of Physical Chemistry A, 2007, Vol. 81, No 10, pp. 1776-1780.

41. Ivanov V.A., Gorshkov V.I., Timofeevskaya V.D., Drozdova N.V., Reactive and Functional Polymers, 1998, Vol. 38, No 2-3, pp. 205-218.

Ivanov Vladimir A. - Doctor of Chemistry, Professor. Department of Chemistry M.V. Lomonosov Moscow State University, Moscow, e-mail: ivanov@phys.chem.msu.ru

Karpyuk Ekaterina A. - Doctor of Chemistry, Dozent. Department of Chemistry M.V. Lomonosov Moscow State University, Moscow

Gavlina Olga T. - Doctor of Chemistry, Senior Scientist. Department of Chemistry M.V. Lomonosov Moscow State University, Moscow

Kargov Sergei I. - Doctor of Chemistry, professor, Department of Chemistry M.V. Lomonosov Moscow State University, Moscow 